\title{
Macro- and micro-scale mixing in a shaken bioreactor for fluids of high viscosity
}

\author{
Gregorio Rodriguez $^{\mathrm{a}}$, Martina Micheletti ${ }^{\mathrm{a}}$, Andrea Ducci ${ }^{\mathrm{b}}$, \\ ${ }^{a}$ Biochemical Engineering Department, University College London, Torrington Place, WC1E \\ 7JE, UK \\ ${ }^{b}$ Mechanical Engineering Department, University College London, Torrington Place, WC1E \\ 7JE, UK
}

\begin{abstract}
Orbitally shaken bioreactors, OSRs, are commonly employed in the pharmaceutical industry at the drug development and screening stage, because they provide a high throughput solution where several cell cultures can be run in parallel. In general cell-culture media used in bioprocessing exhibit a viscosity close to water, but this hypothesis is less valid when high density cell cultures are considered, such as in continuous fermentations, where cells are retained in the bioreactor at large densities and the drug-product is continuously removed and fed to the downstream capture steps of the process. In this context the viscosity of the culture media increases with culture time and with the amount of biomass present in the reactor. In this frame of work two sets of measurements, based on DIMST and pLIF, were carried out to further study the mixing dynamics in a orbitally shaken cylindrical reactor when fluids of viscosity higher than water are considered. These data allowed to identify different flow transitions, which have not been previously observed in the PIV experiments of Ducci and Weheliye [1]. The mixing time measurements highlighted the presence of a poor mixing region for all the flow regimes considered, irrespective of the vortical structure present in the flow, and an attempt was made to quantify the diffusion process occurring at the edges of this region. A more detailed understanding of the flow and deformation dynamics occurring at small scales can
\end{abstract}

${ }^{*}$ Corresponding author $\overline{\text { a.ducci@ucl.ac.uk }}$

Preprint submitted to ChERD Journal

December 21, 2017 
be gained from the pLIF measurements. These allowed to visualise the lamellar structures induced by the flow deformation occurring over several orbital cycles. The growth rate of the material lines was estimated and compared to models commonly employed in the literature to assess mixing dynamics. These information are relevant for micro-mixing models, when the mixing process is limited by the growth of the interfacial area rather than the reaction time.

Keywords: Mixing; Shaken Bioreactor; High viscosity fluids; pLIF.

\section{Introduction}

Orbitally shaken reactors, OSRs, are ubiquitous in cell cultures and bioprocess development of animal or plant cells with low oxygen demand. Small scale OSRs, such as Microtitre plates and/or Erlenmeyer flasks, are commonly employed at the drug development and screening stage because they provide a high throughput technology where several cell cultures can be run in parallel, and are characterised by a well defined free-surface interface and controlled shear conditions [2]. In recent years large orbital single-use bioreactors up to 1000L have proven successful at sustaining cell culture for shear sensitive cell lines. For example the Kühner OrbShake employs the agitation principle of shaken flasks and micro-well plates, providing a unique single-use technology for the entire upstream process, thus facilitating scaling-up and simplifying regulatory approval.

Recent works have provided a better understanding of the flow and mixing characteristics of cylindrical OSRs. At low agitation rate and for fluid of water-like viscosity the flow dynamics in OSRs are characterised by a toroidal vortex inclined below the free surface [3]. Weheliye et al. [3] report that the toroidal vortex expands in size towards the bottom of the vessel and increases in intensity with increasing Froude number (i.e. Froude number, $F r=2 \pi^{2} N^{2} d_{o} / g$ ). When the vortex has reached the size of the tank (critical Froude, $F r_{c r}$ ), a flow transition occurs and the fluid dynamics within the reactor are characterised by an axial vortex precessing around the vessel axis. This transition is also denoted 
by an increasing phase lag between the wave of the fluid free surface and the orbital movement of the reactor (i.e. out-of-phase condition). The scaling law of Weheliye et al. [3] can predict the onset of the flow transition, and depending on the non-dimensional fluid height considered the critical Froude number can be estimated either from Equation $1\left(h / d_{i} \leq\left(d_{o} / d_{i}\right)^{0.5}\right)$ or Equation 2 $\left(h / d_{i} \geq\left(d_{o} / d_{i}\right)^{0.5}\right)$.

$$
\begin{gathered}
F r_{c r}=h\left(d_{o} / d_{i}\right)^{0.5} / a_{o} d_{i} \\
F r_{c r}=1 / a_{o}
\end{gathered}
$$

where the coefficient $a_{0}=1.4$ for water. Most of the works found in the literature on OSRs concern fluids of water-like viscosity, which is in effect a close representation of cell-culture media commonly employed in bioprocessing [4, 5, 6, 7, 8, 9, 10]. This assumption is less valid for high density cell culture, where a 2-3 fold increase in viscosity has been observed with culture time and the biomass increase [11]. Büchs et al. [12] report that an increase in viscosity promotes "out-of-phase" conditions with a significant reduction of oxygen transfer and mixing intensity, and altered cell metabolism. Büchs et al. [12] measured the power consumption for fluids with dynamic viscosity up to $\mu=200 \mathrm{mPa}$ s (cf. water, $\mu=1 \mathrm{mPa}$ ), whilst Kim and Kizito [13] performed numerical simulations and flow visualisations for kinematic viscosity in the range $1 \times 10^{-6} \leq \nu \leq 1.6 \times 10^{-5} \mathrm{~m}^{2} / \mathrm{s}$. Ducci and Weheliye [1] carried out Particle Image Velocimetry (PIV) experiments to investigate the effects of fluid viscosity on the flow in a cylindrical OSR. The linear relationship between the inclination of the free surface wave and the Froude number, was confirmed also for fluid of high viscosity. The constant of proportionality, $a_{o}$, was found to decrease with increasing viscosity according to the power-law of Equation 3 .

$$
a_{o}=a_{o_{w}}\left(\frac{\nu}{\nu_{w}}\right)^{-0.0256}
$$

where $a_{o_{w}}$ is the constant for water (i.e. 1.4), and $\nu_{w}$ and $\nu$ are the kinematic viscosities of water and of the viscous fluid under study, respectively. Ducci and 
Weheliye [1] summarised the different flow dynamics encountered in the shaken 5 reactor in a Fr-Re transition map. For $\nu \leq 1.7 \times 10^{-6} \mathrm{~m}^{2} \mathrm{~s}^{-1}(R e>10000)$ they found that the flow exhibited similar dynamics to those obtained for water with toroidal and axial vortices controlling the flow for in-phase and out-ofphase conditions, respectively. For $\nu \geq 1 \times 10^{-5} \mathrm{~m}^{2} \mathrm{~s}^{-1}(R e<5000)$ the flow dynamics were significantly different: at low $F r$ the flow exhibited a vortex region below the lowest side of the free surface which spread over the bottom of the reactor as $F r$ was increased. At high $F r$, the free surface was wavy and a toroidal vortex rotating in the opposite direction to that reported for water-like viscosity and in-phase conditions, was reported.

Discacciati et al. 14] investigated numerically and experimentally the free15 surface dynamics in high-viscosity fluids ( $\mu=1 \mathrm{~Pa} \mathrm{~s}, 98 \%$ aqueous glycerol solution). They found that a two-dimensional free surface occurred at $F r_{d o}=0.12$, while at $F r_{d o}=0.21$ it was highly three-dimensional. Path-lines of virtual fluid parcels seeded within the reactor were reconstructed from the simulated velocity fields. Fluid parcels were tracked for $8 \mathrm{~s}$ and it was found that their trajectory was characterised by spiral motions for $F r_{d o}<0.15$, while for $F r_{d o}>0.21$, when the free surface was three-dimensional, a more uniform particle dispersion was reached over the time-frame considered.

Mixing dynamics in shaken bioreactors have been investigated in the works of Rodriguez et al. 8, 9] and Tissot et al. [15], where a pH-colorimetric methodology was employed to determine macro-mixing time (Dual Indicator System for Mixing Time, DISMT). At low rotational speed two different mixing zones were identified within the reactor: a zone below the free surface, where mixing was significantly controlled by the toroidal vortex structure, and a slow mixing region close to the bottom of the reactor, where mixing occurred mainly by diffusion. The macro-mixing time was highly dependant on the feed locations, as mixing was found more effective when reagents insertion was made close to the edges of the toroidal vortex [9]. Tan et al. 16] evaluated the effects of viscosity on mixing time in Erlenmeyer flasks. They used aqueous Poly-VinylPyrrolidone, PVP, polymer solutions with dynamic viscosity up to $\mu=38 \mathrm{mPa}$ 
35 s (i.e. $\mu=38 \mu_{w}$ ). It was found that for fixed vessel size and shaking frequency, the PVP solution required 65 seconds to achieve complete mixing, while the same process took approximately 2 seconds in water.

The research carried in this work builds upon previous studies of the research group on the flow and mixing dynamics of OSRs for fluids of water-like

40 viscosity, and aims at providing a thorough understanding of the mixing induced by macro- and micro-scale flow structures when fluids of viscosity greater than water are considered. From this perspective two sets of experiments based on DIMST and pLIF measurements were carried out to identify and characterise poor mixing regions which are diffusion limited, and provide a more detailed understanding of the flow and deformation dynamics occurring at the micro-scales. These information are relevant for micro-mixing models, when the mixing process is limited by the growth of the interfacial area rather than the reaction time.

\section{Methodology}

\section{2.1. Mixing time}

A cylindrical vessel of internal diameter, $d_{i}=100 \mathrm{~mm}$ (full description can be found in [8]), an iCube colour camera and an Led backlight panel were rigidly mounted on a Kühner LS-X orbital shaker. A sketch of the system set up is shown in Figure 1 (a). Mixing time measurements were performed at fixed orbital diameter, $d_{o}=25 \mathrm{~mm}$, and fluid height, $h=32 \mathrm{~mm}$, while the shaken rotational speed, $N$, was varied. The Dual Indicator System for Mixing Time (DISMT) methodology consists in a fast acid-base (hydrochloric acid and sodium hydroxide) reaction in presence of $\mathrm{pH}$ indicators, which causes colour changes in the fluid within the bioreactor once stoichiometric alkali is added 60 to the initially acid solution [17]. The iCube camera recorded an image for each revolution when the bioreactor position was furthest-to-the-left along the circular orbit, $\varphi=0^{\circ}$. The RGB images were analysed using an custom-made Matlab algorithm based on the variation of the green hue to identify the time 
required to achieve homogeneous mixing across the entire vessel volume. A full description of the DISMT methodology and of the algorithm used to evaluate the mixing time can be found in [9] where water-like fluids were investigated. To extend such methodology to fluids of high viscosity, the hydrochloric acid and sodium hydroxide were prepared at a concentration of $0.75 \mathrm{M}$ in aqueous glycerol solutions matching the viscosity and density of the bulk of the fluid.

70 Three fluid viscosities were evaluated, $\sim 2,4$ and 14 times larger than that of water and are referred to in this work as Fluid $A, B$ and $C$, respectively. The fluid properties of the aqueous-glycerol solutions employed are listed in Table 1.

\begin{tabular}{|c|c|c|c|c|c|c|}
\hline \multirow{2}{*}{$\underset{\Xi}{\text { 㙁 }}$} & \multicolumn{2}{|c|}{ Glycerol } & Density & Dynamic viscosity & Kinematic viscosity & $\nu / \nu_{w}$ \\
\cline { 2 - 7 } & $\mathrm{vv} \%$ & $\mathrm{vw} \%$ & $\mathrm{~kg} / \mathrm{L}$ & {$[\mathrm{cP}$ or $\mathrm{mPa} \mathrm{s}]$} & {$\left[\right.$ St or $\left.\mathrm{m}^{2} \mathrm{~s}^{-1}\right]$} & {$[-]$} \\
\hline $\mathrm{A}$ & 20 & 23.97 & 1.0572 & 2.025 & $1.9 \times 10^{-6}$ & 1.9 \\
\hline $\mathrm{B}$ & 40 & 45.68 & 1.1146 & 4.876 & $4.37 \times 10^{-6}$ & 4.37 \\
\hline $\mathrm{C}$ & 60 & 65.43 & 1.1686 & 16.051 & $1.37 \times 10^{-5}$ & 13.7 \\
\hline
\end{tabular}

Table 1: Fluid properties of the acqueous glycerol solutions.

\section{2. $p L I F$}

Planar Laser Induced Fluorescence (pLIF) was employed to investigate the 75 small scale deformation dynamics occurring in cylindrical OSRs. The pliF system comprised a pulsed $532 \mathrm{~nm}$ dual cavity Litron Nano-L 50-100 mJ (Nd:YAG) laser, an optical arm with cylindrical lens and an 8-bit high-speed camera. The arm, camera, mirror and cylindrical vessel were positioned on a large Kühner SR-200X orbital shaker as shown in Figure 1 (b). The cylinder and orbital diameter were $d_{i}=100 \mathrm{~mm}$ and $d_{o}=50 \mathrm{~mm}$, respectively. Inert Rhodamine $6 \mathrm{G}$ dye was employed in this work for its high solubility, chemical stability and high quantum efficiency. The absorption and emission wavelengths were 532 $\mathrm{nm}$ and $560 \mathrm{~nm}$, respectively, while the camera was equipped with a cut-off or- 
ange filter $(540 \mathrm{~nm})$. A Hall-effect sensor was installed on the SR-200X shaker to provide a reference external trigger for each revolution. This was linked to a timing box directly connected to the Dantec Dynamic Studio software which controlled the laser pulses and the camera acquisition. The Rhodamine dye was fed to the system through a syringe pump that could be externally triggered. To be consistent across different experiments dye insertion was made at a phase angle, $\varphi=0^{\circ}$, and the feeding pipe was positioned at the centre of the vessel. Experiments were started after few shaker revolutions to ensure the flow had reached steady state. pLIF experiments were limited to Fluid $C, \nu=1.37 \times 10^{-5}$ $\mathrm{m}^{2} \mathrm{~s}^{-1}$ for $N=80-110 \mathrm{RPM}$ because the diffusion rate of Rhodamine in such a fluid was low, and material lines could be clearly observed.

\section{3. Results and Discussion}

The results and discussion section is organised into two parts, the first focusing on the large scale features of the mixing process, where the DISMT method is employed, and a second one where PLIF is employed to assess local mixing dynamics.

100

A visualisation of the mixing dynamics is discussed in section 3.1 providing a direct comparison of the mixing dynamics occurring in the cylindrical reactor for fluids of increasing viscosity. The estimates of mixing times are provided in section 3.2, where the effect of the fluid viscosity on mixing number is discussed. Section 3.3 investigates the impact of the poor mixing region delimited by the toroidal vortex on the global mixing time. The reduction in size of the poor mixing region due to the diffusion of base is evaluated in section 3.4 employing Fick's $1^{\text {st }}$ law $\left(J=-D \frac{\partial c}{\partial x}\right)$

A detailed analysis of the local deformation dynamics induced by increasing the rotational speed for a high viscosity fluid (Fluid C) is addressed by means of pLIF measurements in section 3.5. An attempt is made to estimate the cyclic increment of a material line by the action of the vortical cells present close to the wall in section 3.6 and how its growth rate can be related to mixing time 
models identified by Meunier and Villermaux [18], which can predict the mixing time from the type of material lines growth. For a scalar blob of dye, $s_{0}$, if the material lines grow linearly, as $\gamma t$, (where $\gamma$ is the rate of deformation), the characteristic time, $t_{s}$ is $t_{s} \sim \gamma^{-1} \mathrm{Pe}^{1 / 3}$ (where $\mathrm{Pe}=\gamma s_{o}^{2} / D$ is a Peclet number, and $D$ the diffusivity). This for example occurs for a point vortex. If the material lines grow as $(\gamma t)^{2}$, then $t_{s} \sim \gamma^{-1} P e^{1 / 5}$, and the material grows in three-dimensions. Finally if the material lines grow exponentially as $e^{\gamma t}$ then ${ }_{120} t_{s}(2 \gamma)^{-1}[18]$.

\subsection{Mixing dynamics in high viscosity fluids}

The mixing dynamics of fluids of increasing viscosity was evaluated employing the DISMT methodology at different operational conditions. The shaken speed was adjusted for each viscosity to obtain a well developed toroidal vortex below the free surface (105-110 RPM). It should be noted that images presented do not necessarily correspond to analogous time instants across the different viscosity conditions investigated, but were solely selected to best visualise significant features occurring during each mixing process. Figure 2 (a-d) and the corresponding video (supplementary file: DISMT_Fluid_A_ 105RPM.avi) refer to the time evolution of the mixing process for Fluid $A$ at $N=105$ RPM $\left(F r_{d o}=0.15, R e=580\right)$. At the start of the experiment (Figure 2 a) the Sodium Hydroxide (in blue) is quickly broken up by the flow. The shear in proximity of the vortical cell edge generates streaklines of locally alkaline concentrations that visualise the boundary of the vortical structure on the left hand side (LHS) of the vessel. Few revolutions (11) later, Figure 2 (b), the alkaline concentration on the right side (RHS) of the vessel delineates a vortical structure, smaller in size, with the outer and inner sides of the vortical cells being alkaline (blue) and acidic (red), respectively. The alkaline fluid present on the outside of the LHS vortex of Figure 2 (b) is transported to the RHS of Figure 2 (c) ten revolutions later, and starts to neutralise locally the predominant acidic solution. Striations are present in the central section of the vessel, indicating slower mixing in this region. After some time, Figure2 (d), the fluid close to the bottom of the vessel 
is well mixed (yellow), whilst the interior of the toroidal vortex remains acidic (red) with local base concentrations at the boundaries on the RHS vortical cell. This specific experiment was run for a very long time, until full mixing was reached, indicating little fluid exchange through the toroidal vortex boundaries. The free surface profile appears to be wavy, implying that the flow is out-ofphase to a certain degree, although the flow structure is very similar to that exhibited by water-like fluids before flow transition, including the direction of rotation of the toroidal vortex (left cell rotating counter-clockwise, CCW, and right cell clockwise, CW). The flow visualisation of Figure2 (a-d) suggests that for $\nu=1.9 \times 10^{-1} \mathrm{~m}^{2} \mathrm{~s}^{-1}$ the toroidal vortices are still present at this speed, $N=105 \mathrm{RPM}$, which is above that predicted by the scaling law [1, 3] for that viscosity $\left(N_{c r}=92 \mathrm{RPM}\right)$. According to other data collected in this work (not shown here) the flow transition seems to occur somewhere between $N=105-120$ RPM.

The time evolution of the mixing process for Fluid $B$ is shown in Figure 2 (e-h) for $N=110 \mathrm{RPM}\left(F r_{d o}=0.17, R e=260\right)$. In this case the free surface profile is three-dimensional (i.e. Figure 2 e), suggesting out-of-phase condition (i.e. after flow transition) and the alkaline feed is broken up into filaments, which initially neutralise the fluid at the sides of the vessel (Figure 2 f). After 50 revolutions, Figure $2(\mathrm{~g})$, the different $\mathrm{pH}$ and colours delineate an inclined toroid below the free surface with elliptical vortical cells on the LHS and RHS side of the OSR. Figure 2 (h) shows that mixing was first achieved in the bulk of the fluid, whilst the toroid behaves as a segregated mixing zone, denoted by a longer time to reach complete $\mathrm{pH}$ homogeneity. An important feature of the flow, which cannot be perceived by the static images, but becomes clear from the supplementary video (file: DISMT_Fluid_B_110RPM.avi), is the direction of rotation of the toroidal vortex. The cells rotate in the opposite direction to that observed for the previous case: CW on the LHS and CCW on the RHS of the vessel.

The DISMT experiment for Fluid $C$ at $N=110$ RPM is shown in Figure 2 (i-1) $\left(F r_{d o}=0.17, R e=84\right)$. This fluid is 14 times more viscous than water and presents significantly different flow regimes than those observed in water. The 
free surface is three-dimensional suggesting an out-of-phase regime. After insertion, the added base is dragged downward through the centre of the vessel (see Figure $2 \mathrm{j}$ ). The toroidal vortex is clearly visible below the free surface rotating CW on the LHS and CCW on the RHS of the vessel (supplementary video:DISMT_Fluid_C_110RPM.avi), and is characterised by poor mixing (highly acidic concentration, red, for a long time). It is worth to stress that a secondary toroidal vortex can be observed in Figure 2 (1) above the main torus; this is also inclined below the free surface and rotates in the opposite direction to the main structure. This secondary toroidal vortex had not been previously reported in the literature.

A visualisation of how the flow and mixing characteristics vary with increasing rotational speed for a high viscous fluid (Fluid C) is provided in Figures 3 (a-l). For a better comparison among the different sets of the data the image sequence already shown in Figures 2 (i-1) for $N=110$ RPM is repeated in Figures 3 (a-d). It is interesting to note how a small increase of the agitation speed to $N=112$ RPM results in a very different flow dynamics (cf. Figures $3 \mathrm{e}-\mathrm{h}$ ). For this speed the sodium hydroxide concentrations, in blue in Figure 3 (e), outlines a CW vortical cell compressed at the vessel walls on the LHS of the vessel, while in Figure 3(f), and in the supplementary video (DISMT_Fluid_C_112RPM.avi), an area of fast moving flow is observed on the RHS of the vessel, below the free surface. Figures $3(\mathrm{~g}-\mathrm{h})$ show a slow mixing region close to the vessel bottom, with features resembling those of a toroidal vortex, acting as a segregated mixing zone and hindering $\mathrm{pH}$ homogenisation. The vortical cell on the left, the fast moving flow on the right and a toroidal structure at the bottom of the vessel had been observed by Ducci and Weheliye [1] where silicon oils, $\nu=10^{-5}$ and $10^{-4} \mathrm{~m}^{2} \mathrm{~s}^{-1}$, were used as working fluids. In their PIV experiments a strong radial flow was observed below the lowest side of the free surface.

The flow transition to an axial vortex occurs at a rotational speed of $N=115$ RPM (see Figure 3 i-1). A 'whirlpool' like structure generates fast mixing at the OSR sides, and a long lasting un-mixed region lingers at the centre of the bioreactor. The three flow dynamics described in Figure 3(a-l) for Fluid $C$ occur 
substantial differences from those observed for water-like fluids.

\subsection{Effects of fluid viscosity on mixing time}

The effect of the fluid viscosity on mixing time is discussed in this section for an agitation range of $N=100-200 \mathrm{RPM}\left(F r_{d o}=0.13-0.56\right)$ and $\nu=1.9-13.7 \times 10^{-6}$ $\mathrm{m}^{2} \mathrm{~s}^{-1}$ (Fluid $A-C$ ). Figure 4 (a) shows the variation of mixing number, $N t_{m}$, with $F r_{d_{o}}$ for the cylindrical $d_{i}=100 \mathrm{~mm}$ OSR for Fluids $A, B$ and $C$. At the lowest $N$ considered very long mixing time are measured for all the viscosities investigated. This is evident in Figure 4 (a), where data points associated to $F r_{d_{o}}<0.15$ for Fluid $A$ and $F r_{d_{o}}<0.17$ for Fluids $B$ and $C$ have mixing numbers in excess of $N t_{m}=300$. The reason for the slow mixing process is due to poor mixing occurring within the toroidal vortex, as it prevents fluid exchange with the bulk of the fluid (see section 3.1). Data associated with Fluid $A$ and Fluid $B$ show a similar variation with $F r$, and tend to plateau for $F r_{d_{o}}>0.27$ (i.e. $N>140 \mathrm{RPM}$ ), while this is delayed for Fluid $C$ with the mixing number levelling for $F r_{d_{o}}>0.37$ (i.e. $N>160 \mathrm{RPM}$ ). Above $F r_{d_{o}}>0.37$ all fluids under investigation reached a nearly constant mixing number $N t_{m} \approx 50$, which is similar to that of fluids of water-like viscosity [8, 9], where the mixing number, $N t_{m}$, decreases with increasing agitation speed and levels to a nearly constant value of $N t_{m} \approx 50$ at $F r \approx 2 F r_{c r}$.

225

The $N t_{m}$ data of Figure 4 (a) are scaled with the critical Froude number in Figure 4 (b), where $F r_{c r}$ is calculated from Equation 1] and the constant of proportionality, $a_{o}$, is obtained from Equation 3 . For reference purposes the shaded area encloses all the non-dimensional mixing times estimated for waterlike fluids for a very large range of operating conditions (cf. Rodriguez et al. [9]). In agreement with the results obtained with water-like viscosity fluids, a plateu is reached at $F r=1.6-1.8 F r_{c r}$. independently to the vortical cell rotating direction, which corroborates that the law of [3] can be extended to fluids of higher viscosity. 
The mixing map in Figure 5(a-b) indicates the local mixing number, $N t_{m_{i j}}$, where indices $i$ and $j$ identify the coordinates of a generic point. The high shear regions surrounding the toroidal vortex achieve a local degree of homogeneity much faster than that of the fluid entrained within. The size of the region associated to slow mixing is larger for Fluid $B$ (Figure 5 a) than for Fluid $C$, Figure 5 (b) although the mixing number of the fluid within the toroid is 1.5 times larger than that obtained for Fluid $B$.

This behaviour is expected as the critical Froude number, $F r_{c r}$, increases with increasing viscosity, and therefore the flow within the vessel is more developed for Fluid $B$ rather than Fluid $C$ at a given speed (i.e. $N=110 \mathrm{RPM}$ ). This also implies that, at a given $N$ the size of the slow mixing region is inversely proportional to the viscosity of the fluid (cf. Figures 5 a and b).

As seen in Figure 5 (a) and (b), the mixing number of the fluid within the toroid can be 3-5 folds that of the remaining fluid. The two regions achieve homogeneity independently at different times. An arbitrary threshold of 1000 was used to discriminate between slow and fast mixing regions. The mixing performances of the two regions are evaluated independently using two mixing parameters: the standard deviation of the normalised green channel, $\sigma_{G^{*}}$ (Figure $5 \mathrm{c}$ ) and the degree of mixedness, $M$ (Figure $5 \mathrm{~d}$ ). The $\sigma_{G^{*}}$ profile of the fast mixing region presents a peak at the beginning of the measurement, indicating a sharp increase of the colour heterogeneity. On the contrary the fluid within the toroidal vortex presents a delayed peak in $\sigma_{G^{*}}$ and for Fluid $C$ this region remains heterogeneous (large $\sigma_{G^{*}}$ ) until the end of the experiment. The degree of mixedness, $M$, in Figure [5 (d) varies from 0 to 1 , as defined by [9] and a similar delay in reaching the value of 1 is observed for the slow mixing region.

\subsection{Diffusion characteristics}

The segregated mixing zone generated by the toroidal vortical structure is more stable for higher viscosity because of the little fluid exchange between the toroid and the bulk of the fluid in the bioreactor vessel. It is observed that 

size as the experiment progresses. It is assumed that such reduction in size is due to the diffusion of the $\mathrm{NaOH}$ (alkali) from the bulk to the interior of the fluid in the toroidal structure. The diffusion from the bulk to the inner toroid is observable via the size reduction of the acidic region within the toroid (see 270 Figure 2 e-h and Figure $3 \mathrm{a}-\mathrm{d}$ ). The vortical structure can be best represented by an elliptical toroid, whose cross section is approximated here by an ellipse. The perimeter of the vortical cell is assumed to be proportional to the surface area of the toroid. The elliptic cross-section of the LHS toroidal cell was measured for Fluid $A$ and $B$ as shown in Figure 6 (a) ( $N=110$ RPM, $d_{o}=100 \mathrm{~mm}, h=32$ Fluorescence measurements reported in this section $\left(d_{o} / d_{i}=h / d_{i}=0.5\right)$. In the following description different flow structures appear as the shaking speed is increased, and therefore a distinction is made between, principal, $P$, secondary, $S$, and tertiary, $T$, vortical structures based on their size. Figures 7 (a-c) show three 
distinctive moments of the mixing process obtained at $N=80 \mathrm{RPM}\left(F r_{d_{o}}=0.18\right.$, supplementary video file: pLIF_80RPM.avi). After addition of the fluorescent dye, the centrifugal force pushes the dye on the free surface towards the vessel walls. The following description will focus on the LHS of the vessel. A large blob of dye can be observed being drawn towards the bottom (Figure 7] a) on a vertical path parallel to the side walls before curling back up CCW towards the free surface (streak ' $a$ ' in the figure). At the same time part of the dye undergoes stretching resulting in a wavy filament (streak ' $b$ ' in the figure). In Figure 7 (b) streak 'a' continues to elongate towards the free surface in a CCW motion. Streak ' $b$ ' appears to be pulled partially upwards towards the surface and partially downwards towards the vessel bottom, resulting into thin striations pattern present next to streak 'a' close to the left wall (see Figure $7 \mathrm{~b}$ and c). Figure 7 (c), 87 revolutions after Figure 7] (a), visualises the main features of the flow at $N=80$ RPM: a principal toroidal vortical cell $\left(P_{L}\right)$, larger in size in the bulk of the fluid, and a secondary $\left(S_{L}\right)$, smaller and located below the free surface close to the vessel walls. Symmetrical vortical cells are present on the RHS, rotating in the opposite direction $\left(P_{R}\right.$ rotating $\mathrm{CW}$ and $\left.S_{R} \mathrm{CCW}\right)$. As the mixing process continues, the injected dye is transported convectively throughout the bioreactor forming homogeneous lamellae with the aqueous glycerol solution. Due to the high deformation at the edges of the vortical cells the lamellae become thinner, the concentration gradient becomes steeper and diffusion becomes dominant.

A very different flow regime can be observed at $N=90 \operatorname{RPM}\left(F r_{d o}=0.23\right.$, supplementary video file: pLIF_90RPM.avi). The dye enters the fluid from the side walls (see Figure $8 \mathrm{a}$ ) and the Rhodamine dye is drawn downwards by the secondary vortical cell, $S_{L}$, and a wavy streak mainly oriented in the $z$-direction is formed. The streaks are observed to further deform due to the simultaneous and complex interaction of three vortices: a primary vortical cell $P_{L}$, a secondary vortical cell $S_{L}$, and a tertiary vortical cell, $T_{L}$, located at the bottom of the reactor (as labelled in Figure $8 \mathrm{c}$ ). This results in a high shear region at the border between the vortices that promotes the formation of lamellar structure 
oriented radially and directed to the vessel's vertical axis with an oscillatory weave in the $z$-direction. Specular vortical structure can be observed on the RHS ( $P_{R}$ and $S_{R}$ in Figure $10 \mathrm{c}$ ), all close to the free surface and oriented at an angle parallel to the free surface.

At $N=100$ RPM $\left(F r_{d o}=0.28\right.$, supplementary video file: pLIF_100RPM. avi $)$ the inserted dye does not spread on the free surface towards the walls, but it is drawn through the centre of the fluid volume (Figure 9 a) under the action of vortical cell $P_{L}$ (see Figure 9 c). The Rhodamine dye reaches the vessel bottom in Figure 9 (b) and then undergoes elongation towards the walls. The main flow structure in the flow are the two vortical cells $P_{L}$ and $P_{R}$, as labelled in Figure 9 (c), which drag the dye through the centre of the OSR, then to the sides along the bottom and upwards along the vessel walls. Significantly, the direction of rotation of the principal vortical cells at these conditions is opposite to that observed at $N=80-85 \mathrm{RPM}$ for $\nu=1.37 \times 10^{-5} \mathrm{~m}^{2} \mathrm{~s}^{-1}$ and water-like viscosity fluids. The periodic striations visible inside the LHS and RHS vortical cell region in Figure 9 (c) are generated by a smaller cell, $S_{L}$, close to the free surface. A vortical cell pair $\left(T_{L}\right.$ and $\left.T_{R}\right)$ is observed at the centre of the vessel, just below the free surface.

Figures 10 (a-c) summarise the flow structures observed from the pLIF im345 ages at $N=80,90$ and $100 \mathrm{RPM}$. For $N=80 \mathrm{RPM}$ principal vortical cells causing an upward stream through the centre of the vessel are observed together with a secondary vortex close to the wall.

At $N=90$ RPM, as shown in Figure 10 (b), vortical cell $S_{L}$ and $S_{R}$ expand significantly and acts to a greater depth below the free surface. As a result $P_{L}$ and $P_{R}$ reduce in size and are positioned just below the free surface. Figure 10 (c) represents the flow observed at $N=100 \mathrm{RPM}$, which presents a large $\mathrm{CW}$ rotating vortical cell on the LHS and a CCW on the RHS $\left(P_{L}\right.$ and $\left.P_{R}\right)$. From the change of rotation direction of the primary vortex it is hypothesized that these are indeed the secondary vortices described at speed of $N=90 \mathrm{RPM}$, which 355 have further increased in size to become the primary vortex. 


\subsection{Growth rate of a material line}

At $N=80-90 \mathrm{RPM}$ the inserted dye is moved on the free surface by the centrifugal force and enters in the bulk of the flow due to the action of the vortical cells close to the vessel walls. Figure11(a) shows in detail the elongation of the dye on the RHS of the vessel at $N=80$ RPM (as labelled in Figure 10 a) over 7 revolutions. After entering the vessel, the fluid parcel undergoes elongation due to the high shear rates at the edges of vortices $P_{R}$ and $S_{R}$. The intensity of the dye, and consequently its concentration, does not vary significantly over the frames under consideration. The diffusion rate $D$ for Rhodamine is $\approx 8 \times 10^{-10} \mathrm{~m}^{2} / \mathrm{s}$, and is several orders of magnitude smaller than the rate of stretching. The length of each weave, which represents the elongation undergone at each revolution, was measured using Matlab and the process is shown in Figure 11] (b), where each colour refers to one complete shaker revolution.

A close up of the Rhodamine dye deformation at $N=80-90$ on the LHS and RHS can be gained in Figure 12, The LHS streak appears to experience fewer folds before being recirculated by the $S_{L}$ vortical cell, suggesting higher vorticity in the region when compared to $N=80 \mathrm{RPM}$. The amplitude of the dye streak folds are wider and the striation thickness lower than that observed 375 at $N=80 \mathrm{RPM}$.

The striations observed are the concentrations of dye present on the plane of measurement, which bisects the bioreactor along its axis. The toroid is acting three-dimensionally in the fluid and elongates and folds the dye in all directions, although motion and shear in the $r-z$ is dominant at $\varphi=0^{\circ}$. The wavy characteristic of the dye streak can be explained based on the findings of Reclari et al. [19], who performed particles tracking simulations in the flow of a cylindrical OSR. It was found that an individual particle moves along a trochoical path with amplitude proportional to the shaking diameter and inversely proportional to the depth from the free surface. It was found in Rodriguez et al. [8] that local concentrations or streaks of $\mathrm{NaOH}$ were following a spiral path around the toroidal vortex, requiring 1.67 shaker revolutions to complete a loop around 
the toroid axis and 6.68 shaker revolutions to completely revolve around the cylindrical vessel. The wavy trajectory of the the Rhodamine observed in the pLIF images dye is likely to be the cross section of the trochoical streaklines observed by Reclari et al. [19], which intersect the measurement plane.

The variation of the striation length growth ratio, $\frac{\ell_{t}-\ell_{o}}{\ell_{o}}$, of the dye material lines with table revolutions is shown in Figure 13 . The growth ratio was averaged for the material lines on the LHS and RHS of the bioreactor vessel. The lengths were measured at the dye streak centreline, for each elongated section (as shown by the different coloured sections in Figures 12 (a-f). The growth ratio of the striation length has a larger slope the higher the $N$. It can be observed that after 5 revolutions, the dye streak at the $N=90 \mathrm{RPM}$ condition has experienced the largest elongation, due to the wider streak folds shown in Figure 12 (e).

In the present case the data in Figure 12 were best-fitted with a parabolic model which, according to Meunier and Villermaux [18], indicates that the complex interaction of the vortical structures is associated to a material line elongation in three-dimensions.

\section{Conclusion}

The mixing dynamics and flow characteristics of fluids of high viscosity in OSRs were evaluated using DISMT and planar Laser Induced Fluorescence. The DISMT experiments were used to identify the main structures in the flow, and to determine the macro-mixing time, the mixing time of segregated regions in the flow and the rate of reduction in size of the slow mixing regions for different fluid viscosities $\left(\nu=1.9 \times 10^{-6}-1.37 \times 10^{-5} \mathrm{~m}^{2} \mathrm{~s}^{-1}\right)$. The use of pLIF allowed to obtain detailed flow visualisations and an estimate of the local growth rate of material lines for a high viscous fluid $\left(\nu=1.37 \times 10^{-5} \mathrm{~m}^{2} \mathrm{~s}^{-1}\right)$.

The DISMT experiments show distinct flow structures for the selected combination of viscosity and agitation rate. At low viscosity and low $N$, The flow is controlled by a toroidal vortex, which convects most of the fluid within the reactor, but also acts as a segregated mixing zone. With increasing $N$ (at constant 
viscosity), the flow transitions to an axial vortex, and fast mixing is achieved at the sides of the vessel. At the intermediate viscosity investigated, Fluid $B$ $\left(\nu=4.37 \times 10^{-6} \mathrm{~m}^{2} \mathrm{~s}^{-1}\right)$, the toroidal vortex is present at low $N$, but is observed to rotate in the opposite direction than that exhibited at lower viscosities. This results in the added base being drawn through the centre of the fluid to the bottom of the vessel just after insertion. Fluid $C\left(\nu=1.37 \times 10^{-5} \mathrm{~m}^{2} \mathrm{~s}^{-1}\right)$ presents at $N=112$ RPM a flow structure not observed with lower viscosity fluid: a vortical cell compressed at the vessel walls on the LHS of the vessel rotating CW and a radial flow on the RHS moving towards the centre of the vessel. Furthermore Fluid $C$ exhibits different flow features (CCW toroidal vortex, radial flow and axial vortex) over a relatively small range of speed, $110<N<115$.

At high agitation rates the mixing number is nearly constant for all viscosities considered, $N t_{m} \simeq 50$. It is noteworthy that the change in rotation of the vortices' rotation, observed to occur with an increase in viscosity, does not affect mixing number. Although CW rotation on the LHS (present in highly viscous fluids) favours the initial distribution of the added base, the segregated mixing inside the toroid is found to offset the rotation advantage. Current mixing data for high viscous fluid shows that the mixing number plateau at $F r=1.6-1.8 F r_{c r}$. This is in agreement with data for water-like viscosities 435 and indicates that the scaling law of Weheliye et al. 3] can be applied to fluids of high viscosity.

At low $N$, the segregated mixing regions caused by the toroidal vortices present higher concentration of the initial $\mathrm{pH}$, resulting in red (acidic) colour, whilst the fluid outside of the toroid undergoes fast mixing. For the same $N$, the segregated region is larger for fluids of lower viscosity, but tends to mix quicker than higher viscosity fluids. The analysis of the mixing dynamics in the segregated zones proved that the base is quickly broken up by the action of the toroidal vortex and reaches homogeneity in the bulk of the fluid. On the contrary the base reaches the interior of the toroid later and homogenizes far 445 slower, increasing the mixing number tenfold. The segregated regions in the toroidal vortices were further studied by means of measuring the rate of decay 
of their size. Due to the limited fluid transfer between the interior of the vortex and the bulk of the fluid the segregated region showed a linear decay, with the more viscous fluid being characterised by a four times slower process. A cell culture practitioner should take into account the effects of viscosity on mixing when evaluating the risk of damage to cells due to gradients of $\mathrm{pH}$, nutrient, oxygen and metabolic products.

Phase-resolved pLIF measurements showed that at low $N$, when the LHS toroidal vortex cell rotates CCW (and the RHS rotates CW) the centrifugal force pushes the dye to the vessel walls and subsequently enters the fluid from the sides under the action of small vortical cells at the sides of the vessel. The interaction between the small vortical cell close to the walls and the larger ones inside the reactor, which extends further down, stretches and folds the dye in a wavy streak which increases in length and wave amplitude at each revolution, before engulfing in the toroid. At higher $N$, when the LHS toroidal vortex cell rotates CW (and the RHS CCW) the dye is dragged in the flow through the centre of the fluid, and it stretched to the bottom and sides of the vessel. The growth rate of the material lines shows that the stretching and folding mechanisms increase with increasing speed and can be modelled with a parabolic function indicating that the complex vortex interaction on the dye generates elongation in three-dimensions.

\section{Acknowledgements}

Financial support from the Engineering and Physical Sciences Research Council of the U.K. for the EngD Centre (grant ref.EP/G034656/1) and from the industrial sponsor Kühner Shaker AG here is acknowledged.

\section{Nomenclature}

\section{Abbreviations \\ CCW Counter-clockwise}




\author{
CW Clockwise \\ 475 LHS Left hand side \\ OSR Orbitally shaken bio-reactor \\ $\mathrm{P}_{L} \quad$ Primary vortical cell, left \\ $\mathrm{P}_{R} \quad$ Primary vortical cell, right \\ pLIF planar Laser Induced Fluorescence \\ ${ }_{480}$ RHS Right hand side \\ $\mathrm{S}_{L} \quad$ Secondary vortical cell, left \\ $\mathrm{S}_{R} \quad$ Secondary vortical cell, right \\ $\mathrm{T}_{L} \quad$ Tertiary vortical cell, left \\ $\mathrm{T}_{R} \quad$ Tertiary vortical cell, right
}

${ }_{485}$ Greek Symbols
$\mu \quad$ Dynamic viscosity, Pa s
$\mu_{w} \quad$ Dynamic viscosity of water, Pa s
$\nu \quad$ Kinematic viscosity, $\mathrm{m}^{2} / \mathrm{s}$
$\nu_{w} \quad$ Kinematic viscosity of water, $\mathrm{m}^{2} / \mathrm{s}$
${ }_{490} \varphi \quad$ Phase angle of the table, ${ }^{\circ}$

\title{
Roman Symbols
}

$\begin{array}{ccl} & a_{o} & \text { Constant of proportionality, - } \\ & a_{o w} \quad \text { Constant of proportionality for water, - } \\ & d_{i} \quad \text { Inner diameter of the cylinder, } \mathrm{m} \\ 495 & d_{o} \quad \text { Orbital diameter, } \mathrm{m}\end{array}$


Fr Froude number, -

$F r_{c} \quad$ Critical/transitional Froude number, -

$g \quad$ Gravitational acceleration, $\mathrm{m} / \mathrm{s}^{2}$

$h \quad$ Fluid height at rest, $\mathrm{m}$

${ }_{500} \quad \ell \quad$ Length of material dye, $\mathrm{m}$

$N \quad$ Shaker agitation speed, $s^{-1}$

$N t_{m} \quad$ Mixing number, -

$p_{v c} \quad$ Perimeter of the vortical cell, $\mathrm{m}$

Re Reynold's Number, -

\section{References}

[1] Ducci, A., Weheliye, W.H.. Orbitally shaken bioreactors - viscosity effects on flow characteristics. AIChE Journal 2014;60(11):3951-3968.

[2] Klöckner, W., Diederichs, S., Büchs, J.. Orbitally shaken single-use bioreactors. Advances in Biochemical Engineering/Biotechnology 2014;138:4560 .

[3] Weheliye, W., Yianneskis, M., Ducci, A.. On the fluid dynamics of shaken bioreactors, flow characterization and transition. AIChE Journal 2013;59(1):334-344.

[4] Büchs, J., Maier, U., Milbradt, C., Zoels, B.. Power consumption in shaking flasks on rotary shaking machines: I. power consumption measurement in unbaffled flasks at low liquid viscosity. Biotechnology and Bioengineering 2000;68(6):589-593.

[5] Maier, U., Büchs, J.. Characterisation of the gas-liquid mass transfer in shaking bioreactors. Biochemical Engineering Journal 2001;7(2):99-106. 
[6] Micheletti, M., Barret, T., Doig, S., Baganz, F., Levy, M., Woodley, J., et al. Fluid mixing in shaken bioreactors: Implications for scale-up predictions from microlitre-scale microbial and mammalian cell cultures. Chem Eng Scien 2006;61:2939-2949.

[7] Zhang, H., Lamping, S.R., Pickering, S.C., Lye, G.J., Shamlou, P.A.. Engineering characterisation of a single well from 24-well and 96-well microtitre plates. Biochemical Engineering Journal 2008;40(1):138-149.

[8] Rodriguez, G., Weheliye, W., Anderlei, T., Micheletti, M., Yianneskis, M., Ducci, A.. Mixing time and kinetic energy measurements in a shaken cylindrical bioreactor. Chemical Engineering Research and Design 2013;91(11):2084-2097.

[9] Rodriguez, G., Anderlei, T., Micheletti, M., Yianneskis, M., Ducci, A.. On the measurement and scaling of mixing time in orbitally shaken bioreactors. Biochemical Engineering Journal 2014;82:10-21.

[10] Rodriguez, G., Anderlei, T., Micheletti, M., Yianneskis, M., Ducci, A.. Appraisal of fluid flow in a shaken bioreactor with conical bottom at different operating conditions. Chemical Engineering Research and Design $2016 ; 108: 186-197$.

[11] Ozturk, S.S.. Engineering challenges in high density cell culture systems. Cytotechnology 1996;22(1-3):3-16.

[12] Büchs, J., Maier, U., Milbradt, C., Zoels, B.. Power consumption in shaking flasks on rotary shaking machines: II. Nondimensional description of specific power consumption and flow regimes in unbaffled flasks at elevated liquid viscosity. Biotechnology and Bioengineering 2000;68(6):594-601.

[13] Kim, H.M., Kizito, J.P.. Stirring free surface flows due to horizontal circulatory oscillation of a partially filled container. Chemical Engineering Communications 2009;196(11):1300-1321. 
[14] Discacciati, M., Hacker, D., Quarteroni, A., Quinodoz, S., Tissot, S., Wurm, F.. Numerical simulation of orbitally shaken viscous fluids with free surface. International Journal for Numerical Methods in Fluids 2013;71(1):294-315.

[15] Tissot, S., Farhat, M., Hacker, D.L., Anderlei, T., Kühner, M., Comninellis, C., et al. Determination of a scale-up factor from mixing time studies in orbitally shaken bioreactors. Biochemical Engineering Journal 2010;52(2):181-186.

555 [16] Tan, R.K., Eberhard, W., Büchs, J.. Measurement and characterization of mixing time in shake flasks. Chemical engineering science 2011;66(3):440447.

[17] Melton, L.A., Lipp, C., Spradling, R., Paulson, K.. Dismt-determination of mixing time through color changes. Chemical Engineering Communications 2002;189(3):322-338.

[18] Meunier, P., Villermaux, E.. How vortices mix. Journal of Fluid Mechanics 2003;476:213-222.

[19] Reclari, M., Dreyer, M., Tissot, S., Obreschkow, D., Wurm, F.M., Farhat, M.. Surface wave dynamics in orbital shaken cylindrical containers. Physics of Fluids (1994-present) 2014;26(5):052104. 


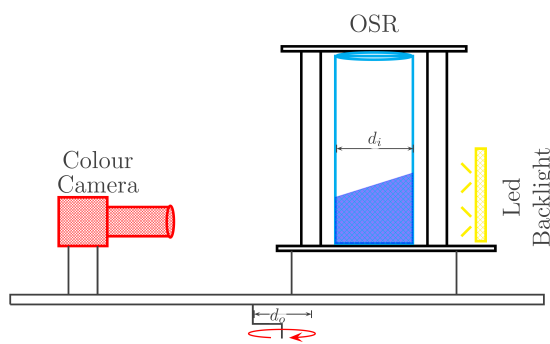

(a)

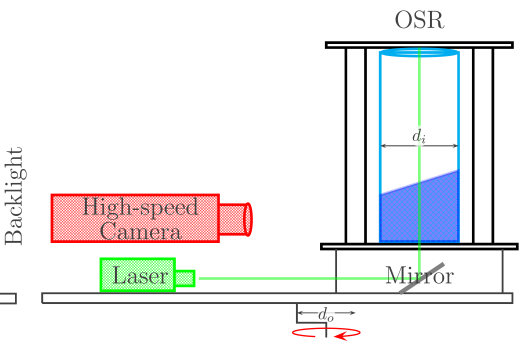

(b)

Figure 1: Diagram of the experimental set-up for (a) mixing time measurements; (b) pLIF.

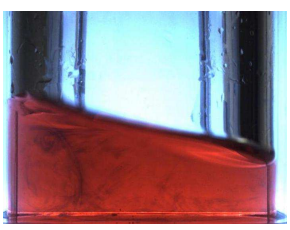

(a)

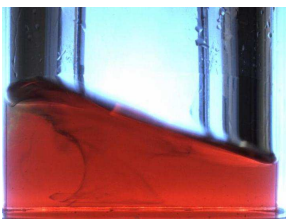

(e)

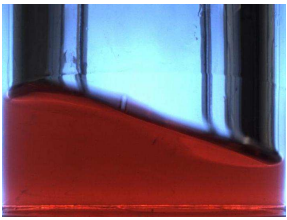

(i)

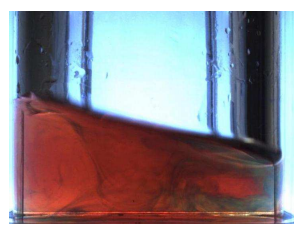

(b)

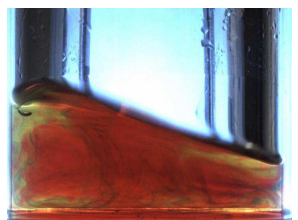

(f)

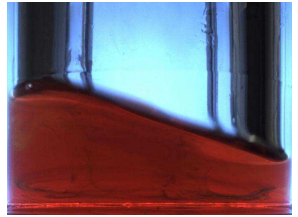

(j)

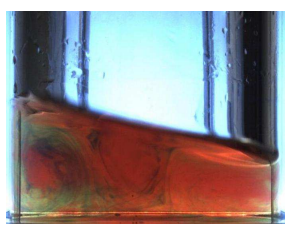

(c)

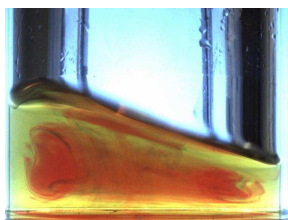

(g)

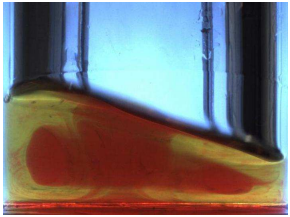

(k)

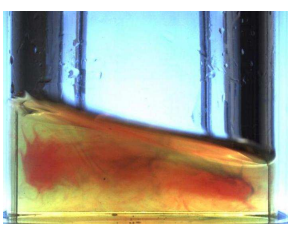

(d)

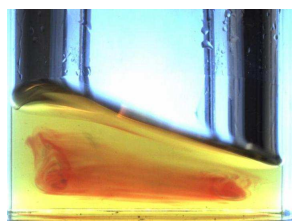

(h)

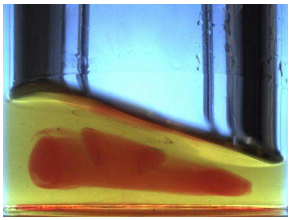

(1)

Figure 2: Time evolution of the mixing dynamics inside the $d_{i}=100 \mathrm{~mm}$ bioreactor for (a-d) Fluid $A, N=105$ RPM; (e-i) Fluid $B, N=110$ RPM; (i-l) Fluid $C, N=110$ RPM; $\left(h / d_{i}=0.32\right.$, $\left.d_{o} / d_{i}=0.25\right)$. 


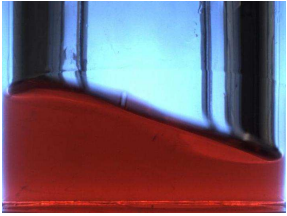

(a)

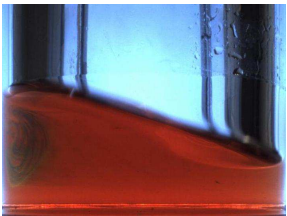

(e)

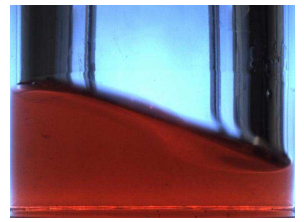

(i)

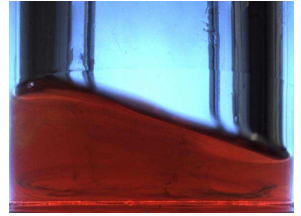

(b)

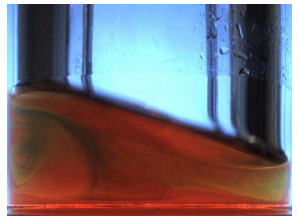

(f)

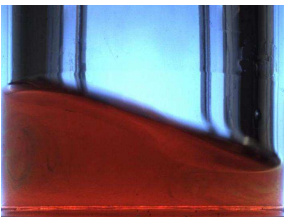

(j)

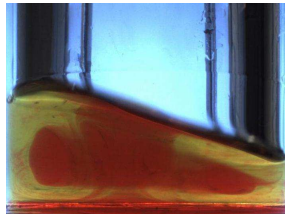

(c)

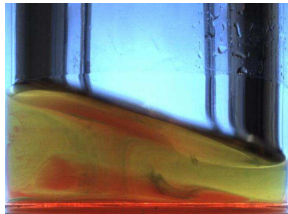

(g)

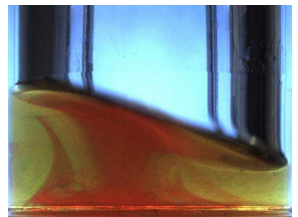

(k)

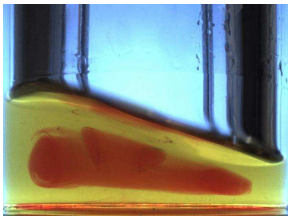

(d)

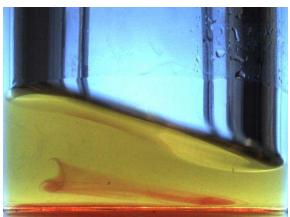

(h)

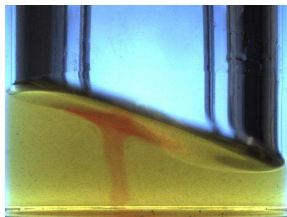

(1)

Figure 3: Time evolution of the mixing dynamics inside the $d_{i}=100 \mathrm{~mm}$ bioreactor for Fluid $C$, for three speeds; (a-d) $N=110$ RPM, (e-h) $N=112$ RPM, (i-l) $N=115$ RPM. $\left(h / d_{i}=0.32\right.$, $\left.d_{o} / d_{i}=0.25\right)$.

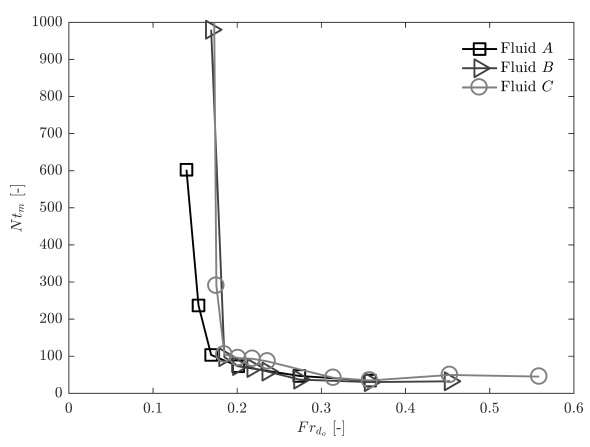

(a)

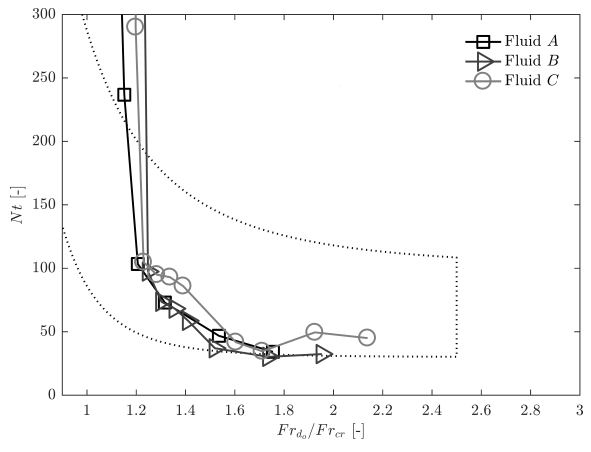

(b)

Figure 4: (a) Variation of mixing number, $N t_{m}$, with increasing Froude for fluids of different viscosity; (b) Variation of mixing number, $N t_{m}$, with $F r_{d_{o}} / F r_{c r}$ for fluids of different viscosity: ( $\square)$ Fluid $A,(\triangleright)$ Fluid $B$ and $(\bigcirc)$ Fluid $C$. Shaded area representing mixing numbers for water-like fluids (see [9]) 


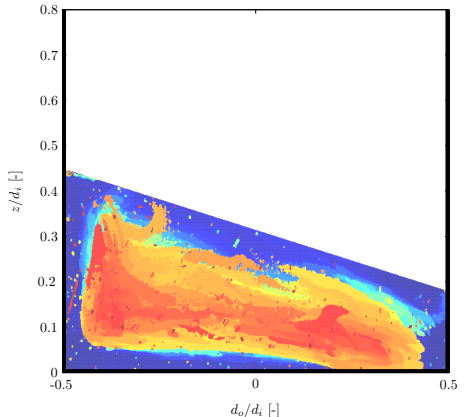

(a)

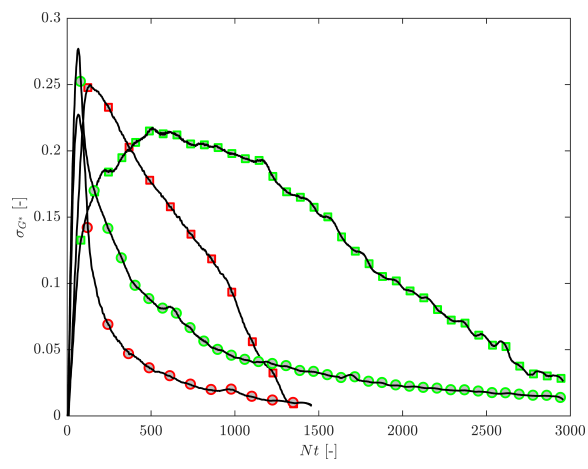

(c)

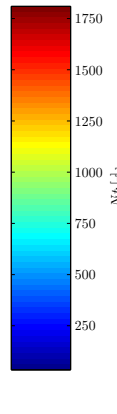

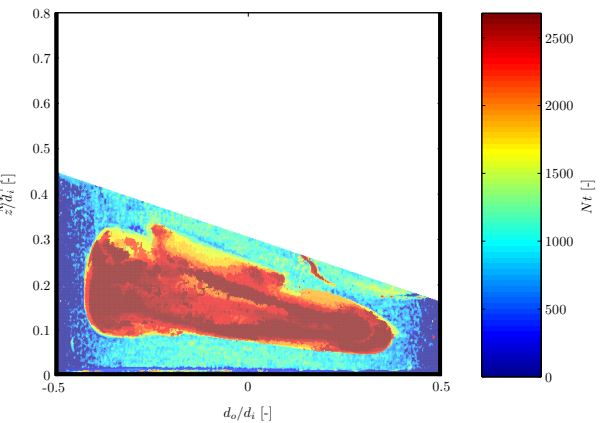

(b)

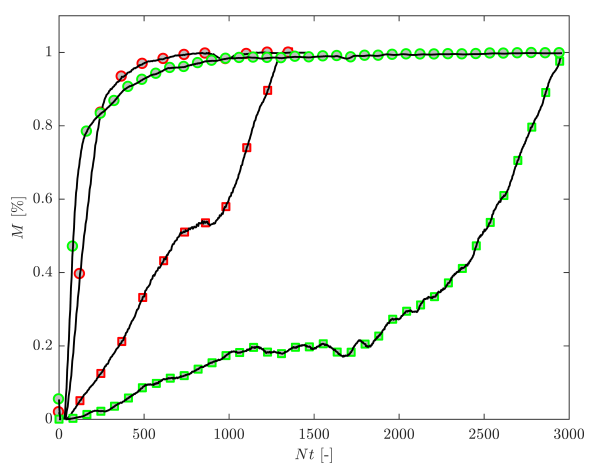

(d)

Figure 5: Mixing time map for bioreactor operating at $N=100$ RPM: (a) Fluid $B$,(b) Fluid $C$; (c) Variation in time of the standard deviation of the Green channel output, $\sigma_{G^{*}}$ and (b) coefficient of mixedness, $M$, in the ( $\square$ ) slow mixing and (o) fast mixing regions, for Fluid B (red) and Fluid C (green). 


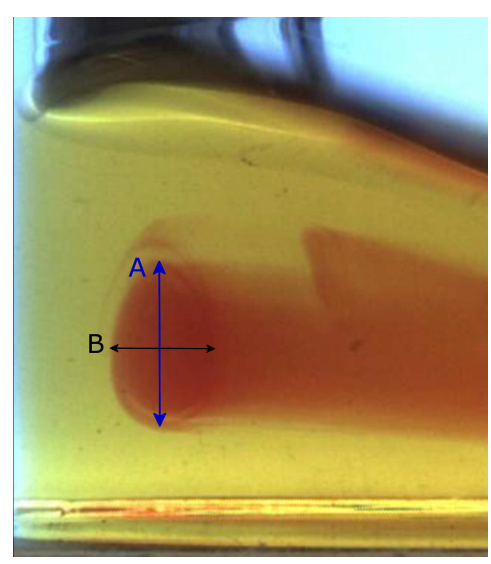

(a)

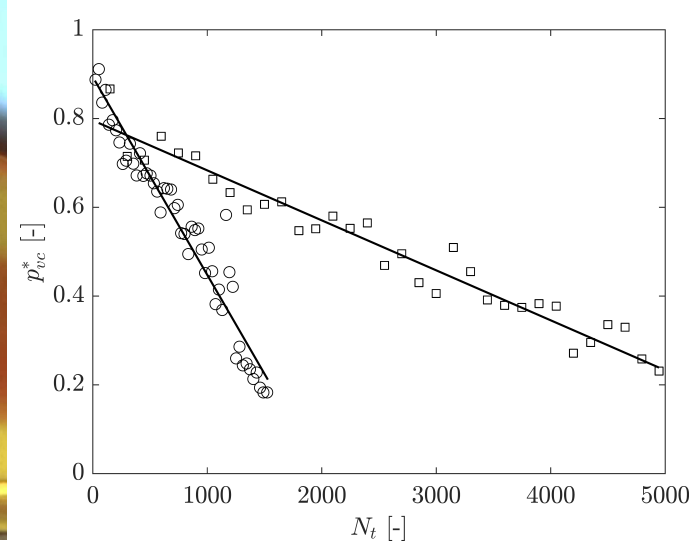

(b)

Figure 6: (a) Visualisation of the major (A) and minor (B) axis of the elliptical cross-section of the LHS vortical cell. (b) Time evolution of the normalised perimeter of the vortical cell with linear fit for: $(\bigcirc)$ Fluid $B$; $(\square)$ Fluid $C$. $\left(d_{o}=100 \mathrm{~mm}, h / d_{i}=0.32, d_{o} / d_{i}=0.25, N=110\right.$ RPM).

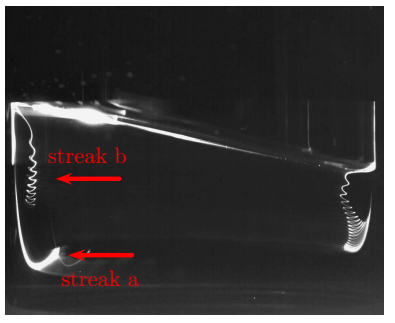

(a)

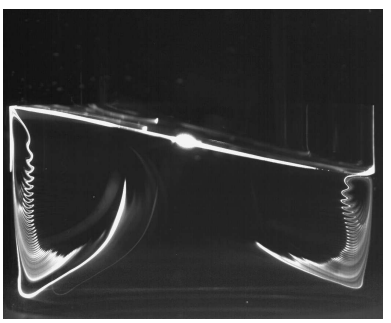

(b)

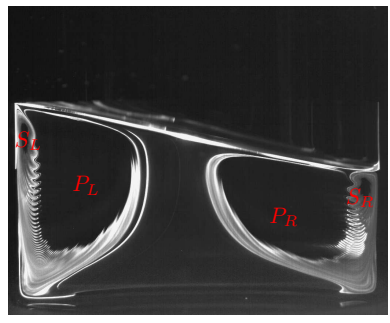

(c)

Figure 7: pLIF images of the cylindrical OSR, for $N=80$ RPM (Fluid $C, h / d_{i}=d_{o} / d_{i}=0.5$ ): (a) $N t=63$, (b) $N t=105$, (c) $N t=150$. 


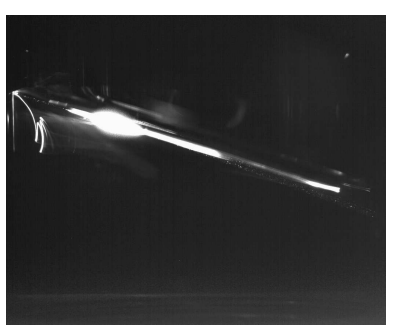

(a)

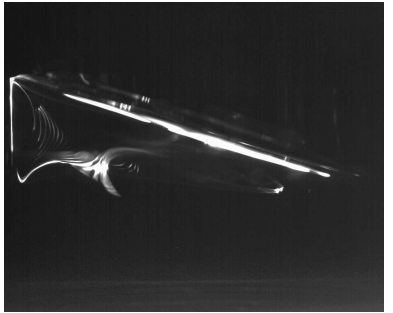

(b)

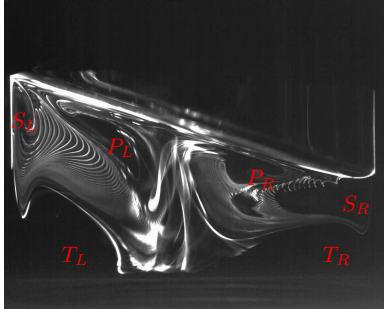

(c)

Figure 8: pLIF images of the cylindrical OSR, for $N=90$ RPM (Fluid $C, h / d_{i}=d_{o} / d_{i}=0.5$ ): (a) $N t=33$, (b) $N t=44$, (c) $N t=108$.

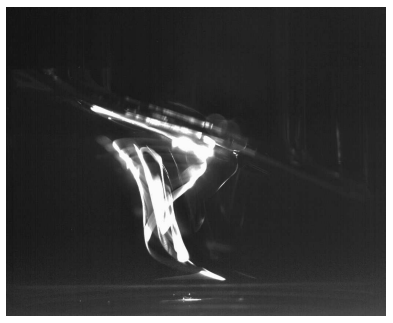

(a)

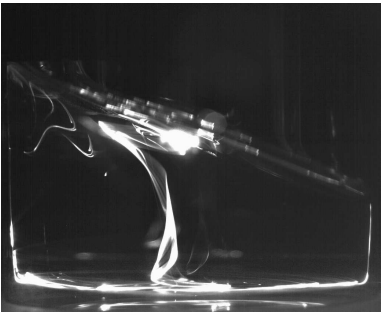

(b)

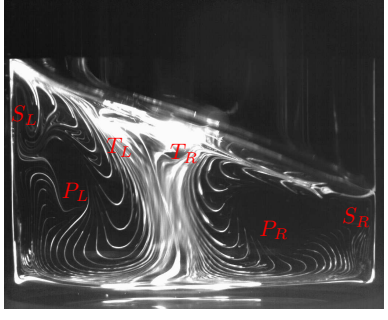

(c)

Figure 9: pLIF images of the cylindrical OSR, for $N=100$ RPM (Fluid $C, h / d_{i}=d_{o} / d_{i}=0.5$ ): (a) $N t=37$, (b) $N t=62$, (c) $N t=93$.

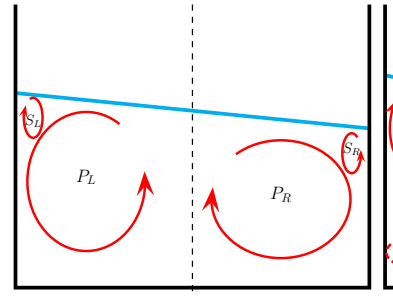

(a)

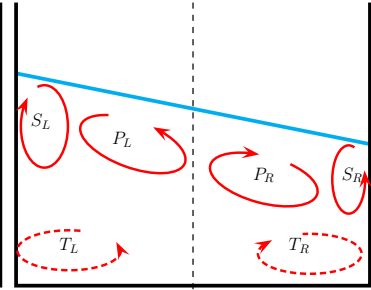

(b)

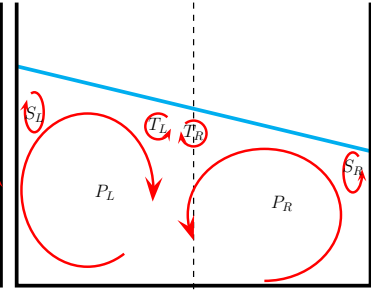

(c)

Figure 10: Schematic diagrams of the flow structures observed from the pLIF images for Fluid $C$ at different $N$ : (a) 80 RPM, (b) 90 RPM, (c) 100 RPM. 


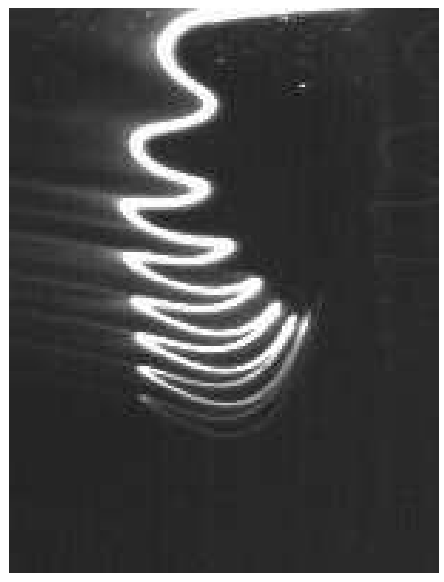

(a)

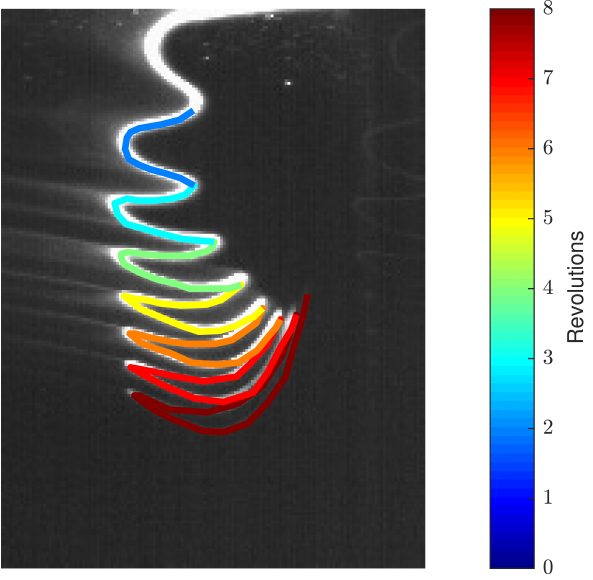

(b)

Figure 11: (a)Detail of the elongation of Rhodamine dye on the RHS of a $d_{i}=100 \mathrm{~mm}$ OSR, Fluid C, $h / d_{i}=0.5, d_{o} / d_{i}=0.5 N=80 \mathrm{RPM}$. (b) Elongation of material lines per shaker revolution, with the colour change indicating each complete revolution. 


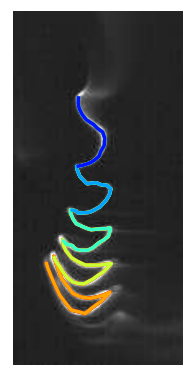

(a)

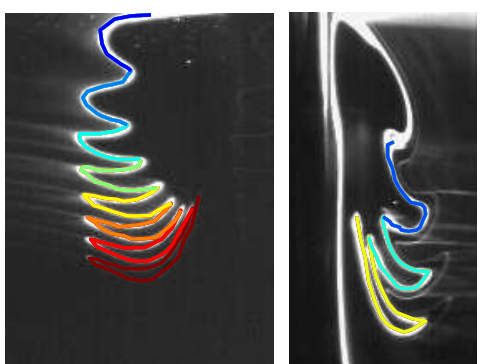

(b)

(c)

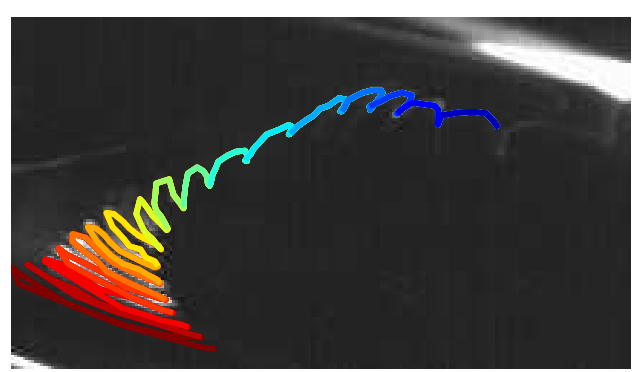

(f)

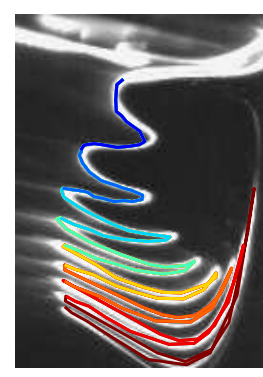

(d)

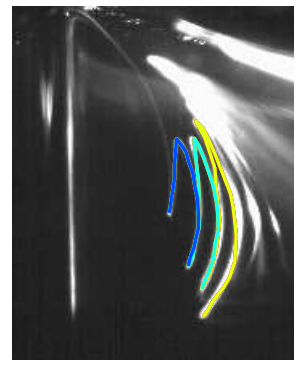

(e)

Figure 12: Detail of the elongation of Rhodamine dye, with the colour change indicating each complete revolution, on the (a) LHS and (b) RHS at N=80 RPM;(c) LHS and (d) RHS at $N=85$ RPM; (e) LHS and (f) RHS at $N=90$ RPM. 


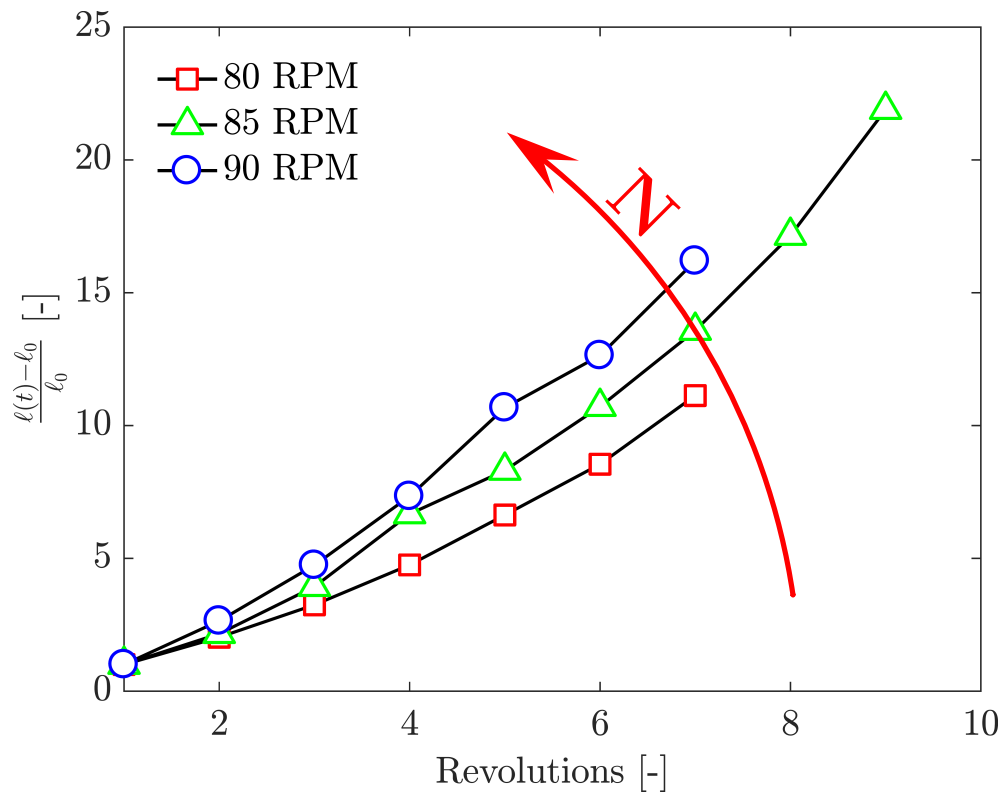

Figure 13: Evolution in time of the material line growth ratio, $\frac{\ell(t)-\ell_{0}}{\ell_{0}}:(\square) N=80 \mathrm{RPM},(\triangle)$ $N=85 \mathrm{RPM}$ and $(\bigcirc) N=90 \mathrm{RPM}$. 